\title{
LATTICES ALL OF WHOSE CONGRUENCES ARE NEUTRAL ${ }^{1}$
}

\author{
CHINTHAYAMMA MALLIAH AND PARAMESHWARA BHATTA, $\mathrm{S}^{2}$
}

\begin{abstract}
We derive a necessary condition for all congruences on a lattice to be neutral, and we show that a stronger condition of the same type characterizes relatively complemented lattices. We also find a condition necessary and sufficient for all congruences to be neutral.
\end{abstract}

1. Introduction. G. Gratzer [4] posed the following problem.

PROBLEM 1.1 (PROBLEM III.7 of [4]). Develop structure theorems for lattices all of whose congruences are standard, distributive or neutral.

This paper gives a solution to this problem for the case of neutral congruences.

First we derive a necessary condition on lattices, for all of whose congruences to be neutral, which is stronger than that given by Iqbalunnisa [5]. Further, using a much stronger condition, a characterization of relatively complemented lattices is obtained. Finally, a complete solution to the last part of the problem is given by another approach.

For basic notations and results we refer the reader to G. Gratzer [4].

\section{Case of neutral congruences.}

THEOREM 2.1. A necessary condition for all congruence relations of a lattice $L$ to be neutral is that $L$ satisfies the following condition.

(C) For $a, b, c, d \in L, a>b, c>d, a / b \approx_{w} c / d$ implies the existence of $c_{1} \in L$, $c \geq c_{1}>d$, such that $c_{1} / d \approx_{w} a / b$.

PROOF. Let all congruence relations of $L$ be neutral and $a / b \approx_{w} c / d, a>b$, $c>d, a, b, c, d \in L$. Then there exists a neutral ideal $I$ of $L$ such that $\Theta(a, b)=\Theta[I]$.

Now $a \equiv b(\Theta[I])$ and $I$ is a standard ideal and hence by a theorem of $[4]$ there exists $i \in I$ such that $a=b \vee i$.

But $i / b \wedge i \nearrow a / b \approx_{w} c / d$ implies $i / b \wedge i \approx_{w} c / d$. Clearly $i, b \wedge i \in I$ and $I$ is a dually distributive ideal. Hence, by a corollary of [2] there exist $a_{1}, b_{1} \in I$ and $c_{1} \in L, c \geq c_{1}>d$, such that $a_{1} / b_{1} \nearrow c_{1} / d$. But as $a_{1}, b_{1} \in I$ and $\Theta[I]=$ $\Theta a / b, a_{1} \equiv b_{1}(\Theta a / b)$, which implies $c_{1} \equiv d(\Theta a / b)$. Hence, there exists a finite sequence of elements $d=d_{1}<d_{2}<\cdots<d_{n}=c_{1}$ such that $d_{i+1} / d_{i} \approx_{w} a / b$ for $i=0,1, \ldots, n-1$. In particular, $d_{2} / d \approx_{w} a / b$, where $d<d_{2} \leq c$, which proves the theorem.

Since (C) implies weak modularity for any lattice $L$, the result of [5] follows immediately. In fact, $(C)$ is stronger than weak modularity, for, even the three element chain does not satisfy $(\mathrm{C})$.

Received by the editors February 29, 1984.

1980 Mathematics Subject Classification. Primary 06B10.

${ }^{1}$ Forms a part of the Doctoral Thesis of Parameshwara Bhatta, S. submitted to the Magnalore University on 20th July 1983.

${ }^{2}$ The research is supported by CSIR, New Delhi, India, under the Research Fellowship Scheme. $0002-9939 / 85 \$ 1.00+\$ .25$ per page 
It is interesting to note that relatively complemented lattices always satisfy (C). In fact, a condition stronger than (C) characterizes these lattices as shown in the following.

THEOREM 2.2. A lattice $L$ is relatively complemented iff $a, b, c, d \in L, b<a$, $d<c, a / b \stackrel{n}{\approx}_{w} c / d$ imply $a / b \stackrel{n}{\approx} c^{\prime} / d$, where $d<c^{\prime} \leq c$.

PROOF. Let $L$ be relatively complemented and let

$$
a / b=e_{0} / f_{0} \sim_{w} e_{1} / f_{1} \sim_{w} \cdots \sim_{w} e_{n} / f_{n}=c / d
$$

The inductive assumption for $n-1$ weak perspectivities implies the existence of $e_{n-1}^{\prime} \in L$ such that

$$
a / b \stackrel{n-1}{\approx} e_{n-1}^{\prime} / f_{n-1}, \quad f_{n-1}<e_{n-1}^{\prime} \leq e_{n-1}
$$

Also, using the assumption for the dual of $L$, there exists $f_{n-1}^{\prime} \in L$ such that $a / b_{n-1} \approx e_{n-1} / f_{n-1}^{\prime}, f_{n-1} \leq f_{n-1}^{\prime}<e_{n-1}$.

Case (1). Suppose $e_{n-1} / f_{n-1} \nearrow_{w} c / d$. Then

$$
a / b \stackrel{n-1}{\approx} e_{n-1}^{\prime} / f_{n-1} \nearrow c^{\prime} / d, \quad \text { where } d<c^{\prime}=e_{n-1}^{\prime} \vee d \leq c .
$$

Hence $a / b \stackrel{n}{\approx} c^{\prime} / d, d<c^{\prime} \leq c$.

Case (2). If $e_{n-1} / f_{n-1} \searrow_{w} c / d$, consider a relative complement $c^{\prime}$ of $c \wedge f_{n-1}^{\prime}$ in $[d, c]$.

Now $e_{n-1} / f_{n-1}^{\prime} \searrow c / c \wedge f_{n-1}^{\prime} \searrow c^{\prime} / d$ and therefore $e_{n-1} / f_{n-1}^{\prime} \searrow c^{\prime} / d$, where $d<c^{\prime} \leq c$.

Hence $a / b \stackrel{n-1}{\approx} e_{n-1} / f_{n-1}^{\prime} \searrow c^{\prime} / d$. Consequently, $a / b \stackrel{n}{\approx} c^{\prime} / d$, where $d<c^{\prime} \leq c$, which proves the necessity part by induction.

Conversely, if $a>c>b, a, b, c \in L$, then clearly $a / c \searrow_{w} a / b$. Hence, there exists $c^{\prime} \in L$ such that $a / c \sim c^{\prime} / b$. Clearly $a / c \searrow c^{\prime} / b$ as $c>b$, which implies $c^{\prime}$ is a relative complement of $c$ in $[b, a]$ and hence the theorem follows.

However, condition (C) is not sufficient for all congruences of a lattice to be neutral as it is already known that a homomorphism kernel of a relatively complemented lattice need not be neutral (see [6]). The next theorem gives a solution to Problem 1.1 by another approach.

THEOREM 2.3. Let $L$ be any lattice. Then the following conditions are equivalent.

(1) All congruences of $L$ are neutral.

(2) $L$ has a zero and satisfies the condition: $x \leq y \vee z, x, y, z \in L$, implies the existence of $a \in L$ such that $a \vee x=(a \wedge y) \vee(a \wedge z) \vee(x \wedge y), a \equiv 0(\Theta(x, x \wedge y))$.

(3) L has a zero and satisfies the condition: $x \leq y \vee z, x, y, z \in L$, implies the existence of $a \in L$ such that $a \vee x=(a \wedge z) \vee(y \wedge(a \vee x)), a \equiv 0(\Theta(x, x \wedge y))$.

ProOF. (1) implies (2). Clearly $L$ must have a zero, for the least congruence $\omega=\Theta[\{0\}]$.

Let $x \leq y \vee z$. Then $\Theta(x, x \wedge y)=\Theta[I]$, where $I$ is a neutral ideal. Since $I$ is standard, by a theorem of $[4]$, there exists $a_{1} \in I$ such that $x=(x \wedge y) \vee a_{1}$. 
Now $a_{1} \leq y \vee z, a_{1} \in I$, and $I$ is a dually distributive ideal. Hence, by a corollary of $[\mathbf{1}]$, there exists an $a \in I$ such that $a_{1} \leq a=(a \wedge y) \vee(a \wedge z)$. But then

$$
a \vee(x \wedge y)=\left(a \vee a_{1}\right) \vee(x \wedge y)=a \vee\left(a_{1} \vee(x \wedge y)\right)=a \vee x
$$

Thus

$$
a \vee x=a \vee(x \wedge y)=(a \wedge y) \vee(a \wedge z) \vee(x \wedge y)
$$

Also $a \in I$, and therefore, $a \equiv 0(\Theta(x, x \wedge y))$, which proves (2).

(2) implies (3). From (2), given $x, y, z \in L, x \leq y \vee z$, there exists $a \in L$ such that $a \equiv 0(\Theta(x, x \wedge y))$ and $a \vee x=(a \wedge y) \vee(a \wedge z) \vee(x \wedge y)$.

Now

$$
a \vee x \geq(a \wedge z) \vee(y \wedge(a \vee x)) \geq(a \wedge y) \vee(a \wedge z) \vee(x \wedge y)=a \vee x
$$

Hence (3) follows.

(3) implies (1). Let $x, y \in L, x \geq y$. Then $x=y \vee x$. So by (3) with $z=x$ there exists an $a \in L$ such that $a \vee x=(a \wedge x) \vee(y \wedge(a \vee x)), a \equiv 0(\Theta(x, x \wedge y))$. But this implies $x=a \vee y$ with $a \equiv 0(\Theta x / y)$. Hence, by a remark of [3], all congruence relations of $L$ are standard.

Now it suffices to show that all standard ideals of $L$ are neutral. Let $I$ be a standard ideal of $L$ and $x \leq y \vee z, x \in I, y, z \in L$. Then by (3), there exits $b \in L$ such that $b \vee x=(b \wedge z) \vee(y \wedge(b \vee x)), b \equiv 0(\Theta(x, x \wedge y))$. Since $x, x \wedge y, 0 \in I$ and $I$ is a homomorphism kernel, we get $b \in I$. Hence $b \vee x \in I$.

Further

$$
((b \vee x) \wedge y) \vee((b \vee x) \wedge z) \geq(b \wedge z) \vee(y \wedge(b \vee x))=b \vee x
$$

Hence

$$
b \vee x=((b \vee x) \wedge y) \vee((b \vee x) \wedge z)
$$

Puting $a=b \vee x$, we get $x \leq a=(a \wedge y) \vee(a \wedge z)$ with $a \in I$. Thus, by a corollary of $[2], I$ is a dually distributive ideal, which completes the proof.

\section{REFERENCES}

1. Chinthayamma Malliah and Parameshwara Bhatta, S., Generalizations of dually distributive and neutral ideals to convex sublattices. I, Notre Dame J. Formal Logic (submitted).

2. __ Generalizations of dually distributive and neutral ideals to convex sublattices. II, Notre Dame J. Formal Logic (submitted).

3.

4. G. Gratzer, General lattice theory, Birkhauser Verlag, Basel and Stuttgart, 1978.

5. Iqbalunnisa, Normal, simple and neutral congruences on lattices, Illinois J. Math. 10 (1966), 227234.

6. M. F. Janowitz, A characterization of standard ideals, Acta Math. Acad. Sci. Hungar. 16 (1965), 289-301.

DePARTMENT OF MATHEMATICS, MAgNAlore UNIVERSity, MANGalagangotri, D.K., KARNATAKA, INDIA 